\title{
EDITORIAL
}

\section{More Terminological Blunders}

\section{Stephen E. Braude}

https://doi.org/10.31275/20201899

Creative Commons License CC-BY-NC

In my previous Editorial, I took a short detour

from the main topic (telepathy and mental privacy) to comment briefly on one of the deeper flaws in the trendy, but seriously misguided, practice of replacing the terms "ESP" and "PK" with (respectively) "anomalous cognition" and "anomalous perturbation." As I've discussed in

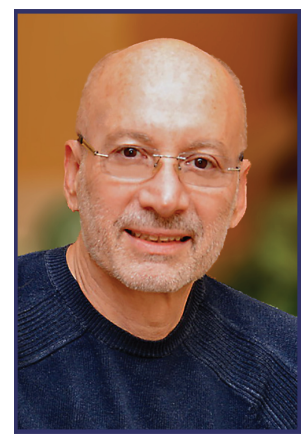
great detail elsewhere (Braude, 2020), there's actually quite a lot that's wrong with this terminological folly. And it's hardly the only time psi researchers have botched efforts to explicate or replace some of the field's key concepts.

The terminological error that I discussed in my earlier Editorial was the failure to accommodate the valuable distinction between ESPcognition and ESP-interaction. And in that Editorial, I also noted that another, and increasingly trendy, practice likewise commits this error. It's the strategy of abandoning the venerable arsenal of psi-terms and replacing them with a single expression-either "nonlocal awareness" or "nonlocal consciousness." (The underlying rationale for this is usually that the traditional vocabulary is likely to be professionally toxic.) I'm thinking about these matters again because recent events have conspired to remind me of still another, but less trendy and prevalent, approach to parapsychological terminology that also deserves a few words of disapproval.

The approach in question tries to explicate "ESP" and "PK" by considering paranormal influence to be a form of action at a distance. Now although the serious parapsychological literature hasn't recently devoted much attention to this strategy, I still encounter it in conversations with people who (for the most part) have only a casual acquaintance with the evidence and issues of parapsychology. But this 
error hasn't been restricted to parapsychological dilettantes. The most prominent, and perhaps the only fairly recent, parapsychologically well-informed exponent of this strategy has been David Ray Griffin, an admirably serious person, who argued for his revisionary terminology in an interesting paper presenting a Whiteheadian approach to parapsychology (Griffin, 1993), and in an otherwise thoughtful book mostly on the evidence pertaining to survival (Griffin, 1997). I'll assume the later work presents the more refined version of his position and so I'll focus on that. In any case, the notion that psi phenomena are all fundamentally instances of action at a distance is not yet dead (even if it can't be said to be thriving). But perhaps I can finally hasten its thorough demise.

In my comments last issue on the abuse of the term "nonlocal," I noted that many apparent examples of both ESP and PK seem clearly to be flagrantly local. If psychic healers can diagnose subtle medical conditions of the client seated before them, that would be an instance of ostensible ESP confined to that small region of space. Similarly, a successful Ganzfeld experiment must be explained in terms of events occurring within the confines of the lab. And we know exactly where telepathic influence is occurring when an experimenter's hypnotic command to fall asleep causes the person in a nearby building suddenly to fall asleep, or where causal influence is occurring when a medium levitates a table. Dubbing such interactions nonlocal serves no usefulor for that matter, intelligible-purpose.

Predictably, those who consider psi phenomena to be forms of action at a distance likewise confront problems about event-locality. Let's note first that Griffin states explicitly that "what is distinctive of the category of the paranormal is the idea of influence at a distance to or from minds (1997, p. 16, italics in original). Thus, PK (or what Griffin termed "expressive psi" in his earlier work) "involves the exertion of causal influence at a distance by a mind" (1997, p. 16, italics in original). And ESP (or what Griffin previously termed "receptive psi") "involves a mind's reception of influence from a distance (p. 16). In both cases Griffin distinguishes these forms of paranormal causation from more ordinary causality-e.g., the brain affecting its own body, or ordinary sensory perception, all seemingly easily explicable in terms of spatiotemporally contiguous and transitive causal links.

So the distance mentioned in "action at a distance" may actually be quite small. What matters is that, in paranormal causation, the 
proximate cause is not spatiotemporally contiguous with its immediate effect. Still, I would have thought it's clear that this can't be right. Consider first the claim that PK is a form of action at a distance. Unfortunately, that view seems clearly to exclude a very familiar form of ostensible materialization, in which ectoplasm emanates from parts of the medium's body. Assuming the phenomenon is genuine, that alone should be enough to consign the view to the trash heap. But equally seriously, it rules out the possibility of PK on one's own body. For example, if PK is always action at a distance, then what we regard as psychic healing would presumably count as PK when the healer's thoughts produced a change directly in another person's body, but it wouldn't be PK if healers healed themselves by this method. Similarly, we wouldn't be able, in principle, even to entertain the hypothesis that the occasionally dramatic physiological effects of self-hypnosis (say, in cases of dissociative identity disorder or multiple personality) are manifestations of PK. But I would have thought it's an interesting, open, and at least partly an empirical question whether somatic manifestations of hypnotic suggestion (and kindred phenomena such as placebo effects) should be classified (along with object movements, materializations, etc.) as PK, or whether they result from quite familiar (though as yet undetermined) contiguous physiological processes.

Incidentally, a similar terminological sin concerns a subset of PK phenomena—namely, what some have called "bio-PK." Evidently, some believe that it's better to eschew the professionally perilous term "PK" and label this subset "DMILS," which we're told stands for "distant influence on living systems." But clearly, "DMILS" can't be regarded as synonymous or coextensive with "bio-PK" because that term, in principle at least, allows for the possibility of non-distant influence on one's own body. Some have even suggested that ordinary volition involves the psychokinetic action of one's mind on one's brain, and (as I noted above) others have proposed that (for all we know) PK might be operative in placebo effects, self-healing, and more familiar hypnotic effects on one's own body (e.g., raising welts on one's skin).

Applying the principle of charity, one might think that there's some way of understanding the phrase "action at a distance" so that it covers instances of PK on one's own body. If so, then unless the concept of distance has lost all conventional meaning, my guess is that "action at a distance" would have to mean something like "action by means of a noncontiguous connection between cause and effect." This rendering 
of the phrase at least has the virtue of complying with Griffin's desire to repudiate billiard-ball causality (roughly, causality by contact) as the model for causal relations generally. However, in his book Griffin seems to reject this option-or so it appears. In earlier and more impetuous essays, he was clearer on the matter. But in his book, he seems to allow for the possibility of at least some PK on oneself. He assumes that "all psychosomatic influences may be mediated through the brain" (p. 144) and that the action of the brain on one's body is not paranormal. And presumably, that's because a full account of how the brain affects the body in such cases would reveal a transitive series of spatiotemporally contiguous causal links. Curiously, however, he also claims that stigmata would involve action at a distance if they're cases of "direct action of the mind on some portion of the body other than the brain" (p. 144).

Frankly, I don't get this. Quite apart from the peculiarity of using the term "distance" in such a case, I don't see why the direct action of mind on the brain is not PK but direct action of mind on the skin would be. Both cases seem prima facie to be examples of mind directly influencing the body. But I'm not concerned here with trying to unravel fine details of Griffin's idiosyncratic view, because there's an overriding flaw in the very idea that PK is always action at a distance. It's a serious error to make a major ontological distinction on the basis of nothing more than a fortuitous spatial arrangement of physical objects. It's analogous to saying that I can hit (or criticize) another person, or make another person unhappy, but I can't hit (criticize) myself, or make myself unhappy, because those forms of causation can only occur over a distance. The proper response to such a claim (after the obligatory guffaw) would be that the processes are the same whether I or another object are affected, and that we shouldn't make such a big deal out of nothing more than a contingent difference in the location of the affected object. Similarly, one can stab, observe, poison, anger, compliment, and listen to both oneself and others-and of course, pat oneself on the back. And indeed, many causal (and epistemological) relations can be reflexive.

Similar problems afflict the attempt to treat ESP as a form of action at a distance. Griffin claims that the term "receptive psi" "covers everything that is usually covered under 'extrasensory perception." But in fact, that approach excludes the possibility of using ESP on one's own body. Presumably, it would be a case of receptive psi when healers 
clairvoyantly diagnose an illness in another person (e.g., to determine the precise location of a blood clot or tumor), but not when healers clairvoyantly elicit the same information concerning themselves. And that does seem as arbitrary and antecedently incredible as maintaining that one can smell another person but not smell oneself, or that one can hear the growling stomach of another person but not one's own.

Interestingly, the escape route that might have worked in the case of PK doesn't work here. It won't help to reconstrue "action at a distance" so that "receptive psi" means something like "the experiencing or registering of causal influences by means of a noncontiguous connection between cause and effect." That move would have consequences nearly as awkward as those they're intended to avoid. Most notably, memory would count as a form of ESP, since (contrary to the popular wisdom) so-called "mnemonic causation" is not explicable in terms of spatiotemporally contiguous causal chains-in particular, with reference to memory traces, which in theory are supposed to bridge the spatiotemporal gap between the present memory event and the temporally remote thing remembered. But as I and others have argued, that view is simply, and quite literally, a form of disguised deep nonsense (Braude, 2002, 2014; Bursen, 1978; Heil, 1978).

Moreover, there's another respect in which the terms "expressive psi" and "receptive psi" fail as synonyms for "PK" and "ESP" (respectively), or simply as conceptually illuminating replacements for those terms. Telepathic influence, of one mind directly on another, is not receptive psi to a telepathic agent. It's receptive psi only to a telepathic percipient (or victim). From the point of view of the agent initiating that influence, it could legitimately be called "expressive psi"-and thus count for Griffin as an instance of PK, even though the causality may not produce a physical effect. But the traditional, venerable, and as far as I can determine still reasonable, view is that telepathy is a form of direct mind-to-mind interaction, irrespective of whether we're considering the point of view of agent or percipient. Granted, it may be difficult (if not impossible) to know whether a particular case is one of telepathic influence on the percipient's mind or PK on the percipient's brain. But no such ambiguity afflicts the concepts of telepathy and PK. It's telepathy so long as the interaction is between minds, and it's PK if the causality is the mind acting directly on a physical object (including the brain). Those two forms of paranormal interaction may be operationally indistinguishable (at least at our present level of technological 
sophistication), but they're easy to distinguish conceptually. So Griffin's view awkwardly and implausibly dissolves (or at least clouds) the otherwise reasonable and easily intelligible distinction between telepathic influence and PK.

Incidentally, in actual cases of ostensible telepathy it's likewise a challenge to determine the direction of the telepathic causal arrowthat is, whether A's mind is imposing its influence on that of $B$, or whether $B$ is somehow aware of (or "reading") the contents of A's mind (as it were, invading A's supposedly private mental space). But the term "receptive psi" seems to cover only the latter option. The former option-A paranormally influencing the thoughts of $B$-would (as before) seem to be an instance of expressive psi and thereby count as a form of PK, even if the interaction is directly mind-to-mind. But there was never anything wrong with the old tradition of considering either causal scenario to be telepathic as long as the causality is strictly between minds or mental states. Nothing here needed fixing! So once again, it's clear that "receptive psi" and "expressive psi" fail either as synonyms or illuminating replacements for (respectively) "ESP" and "PK."

I don't know why parapsychological dilettantes hold the view that psi phenomena are all forms of action at a distance. But Griffin, apparently, is led to this unfortunate position because of the great importance he attaches, right from the start, to the significance of action at a distance for the scientific view of the world. It was simply a mistake to place so much importance on the concept of distance. Griffin is justified in noting that science has occasionally relied on mistaken assumptions about the nature of causal relations. And he and many other philosophers have correctly noted that there's nothing inherently wrong with gappy causation. But the traditional (and by no means universal) insistence on billiard-ball causality, or spatiotemporal contiguity of cause and effect, is merely a symptom of a deeper errornamely, a misguided mechanistic approach to observable phenomena generally, and an associated reliance on what I've called the small-isbeautiful assumption. However, that's a huge topic, far exceeding the appropriate limits for an Editorial. It's also a topic l've covered at length elsewhere (Braude, 1997, 2014).

However, there's still more to say about definitions in parapsychology, and (barring some unforeseen distraction) I'll continue with that topic in my next Editorial. 


\section{REFERENCES}

Braude, S. E. (1997). The limits of influence: Psychokinesis and the philosophy of science (Rev. ed.). University Press of America.

Braude, S. E. (2002). ESP and psychokinesis: A philosophical examination (Rev. Ed.). Brown Walker.

Braude, S. E. (2014). Crimes of reason: On mind, nature, ex the paranormal. Rowman \& Littlefield.

Braude, S. E. (2020). Dangerous pursuits: Mediumship, mind, and music. Anomalist Books.

Bursen, H. A. (1978). Dismantling the machine theories of memory. D. Reidel.

Griffin, D. R. (1993, July). Parapsychology and philosophy: A Whiteheadian postmodern perspective. Journal of the American Society for Psychical Research, 87(3), 217-288. http://www.anthonyflood.com/griffinparapsychology.htm

Griffin, D. R. (1997). Parapsychology, philosophy, and spirituality: A postmodern exploration. State University of New York Press.

Heil, J. (1978, March). Traces of things past. Philosophy of Science, 45, 60-72. https://doi.org/10.1086/288779 